\title{
An in silico approach to map the binding site of doxorubicin on hemoglobin
}

\author{
Shahper Nazeer Khan ${ }^{1}$ and Asad Ullah $\operatorname{Khan}^{1,2, *}$ \\ ${ }^{1}$ Interdisciplinary Biotechnology Unit, Aligarh Muslim University, Aligarh-202002, India; ${ }^{2}$ Bioinformatics distributed Information sub-centre, \\ AMU, Aligarh-202002, India; Asad Ullah Khan* - E-mail: huzzi99@hotmail.com; Phone: 91571 2723088; Fax: 91571 2721776; * Corresponding \\ author
}

received March 08, 2008; revised April 18, 2008; accepted April 22, 2008; published July 14, 2008

\begin{abstract}
:
Binding modalities of doxorubicin (DOX), a widely used antineoplastic anthracyline antibiotic with hemoglobin (Hb) have been studied. The protein and the ligand were prepared using CORINA and protonated with insight II. The best conformation was sought by employing GOLDV. Molecular modeling calculations showed that DOX binds Hb to a non-classical drug binding site. The alpha subunit of $\mathrm{Hb}$ has been assigned to posses the binding site for DOX with a binding affinity $(\mathrm{Ka})=16.98 \times 10^{3} \mathrm{~mol}^{-1}$. The interaction was found to be thermodynamically favorable $\left(\Delta \mathrm{G}^{\circ}=-66.23 \mathrm{KJmol}^{-1}\right)$. The analysis of DOX binding site to Hb suggested that the types of interactions that contribute in this binding are hydrophobic contacts, hydrogen bonding and electrostatic interactions.
\end{abstract}

Keywords: hemoglobin; doxorubicin; molecular docking; binding site

\section{Background:}

It is well established that free molecules of drug can act at the target site. This is because free drugs are rapidly distributed into tissues, which facilitate direct binding to tissue proteins. The binding of drugs to plasma and tissue proteins is common. This is considered to be an important factor to determine the pharmacokinetics and pharmacodynamics of drugs. The formation of drug-protein adducts can cause cellular and tissue toxicity which may be either intrinsic or idiosyncratic in nature $[1,2]$. Protein-drug interactions play an important role in a variety of biological processes and disease treatments. The studies on this aspect may provide information of the structural features of protein that may determine the therapeutic effectiveness of drugs, and become an important research in biomedicine. A high binding affinity for protein has been observed for drugs possessing acidic or strong electronegative functional groups [3], which can bind to more than one binding site with different specificity. It is worth mentioning that saturable binding of some ligands to protein induce alterations in the structure and function of protein. However, selective binding displayed by different ligands may result an allosteric effects, whereby, binding of ligand $\mathrm{A}$ at certain site causes a conformational change in the protein so that binding of ligand $\mathrm{B}$ at a different site is altered [4].

Hemoglobin $(\mathrm{Hb})$ is the iron containing oxygen transport metallo-protein in the red blood cells which is also known for the transport of carbon dioxide and regulation of blood $\mathrm{pH}$ [5]. Each subunit of $\mathrm{Hb}$ is a globular protein with an embedded heme group. The protein tetramer comprises of two $\alpha$ - and two $\beta$-chains assembled to form symmetrical $(\alpha \beta)$ dimers. In the center of each heme group is a $\mathrm{Fe}^{2+}$. The $\mathrm{Hb}$ exists in

ISSN 0973-2063

Bioinformation 2(9): 401-404 (2008) functionally important two isomeric forms the $\mathrm{R}$ form (oxygen/ligand bound state) and the T form (Deoxy state) [6, 7], which helps in proper coordination between upload and release of oxygen.

Doxorubicin (DOX) is an effective chemotherapeutic agent for the treatment of breast cancer, malignant lymphomas, soft tissue sarcoma and various neoplastic diseases. This anthracycline antibiotic has a water-soluble basic amino sugar, daunosamine, which is linked glycosidically to carbon 7 of a four-ringed water insoluble aglycone known as doxorubicinone. Aglycone is a substituted naphthacene quinone with a methoxy group at the 4position and a hydroxyl group plus a hydroxyacetyl group at carbon 9 [8]. DOX has been shown to oxidize $\mathrm{Hb}$ [9] and produce superoxide in red cells [10]. This may result in haemolysis [11]. Although it has been suggested DOX on reduction, reacts directly with oxy-Hb [12]. Its binding site has not yet reported. An array of in solution techniques are available to determine various binding attributes of drugs, which closely represents the physiological conditions, but unable to determine the location of binding sites. The computational modeling was used to identify the amino acids involved in binding site. Moreover, it can also predict various conformational states of bio-molecules such as the oxygen bound form of $\mathrm{Hb}$ as described in the present study.

\section{Methodology:}

Genetic algorithm

Genetic algorithm (GA) is a computer program that mimics the process of evolution by manipulating a collection of datastructures called chromosomes. Each of these encodes a possible 
solution (in terms of a possible ligand-receptor interaction) to the docking problem and may be assigned fitness score based on the relative merit of the solution. The GA utilizes a novel representation of the docking process. Each chromosome encodes an internal conformation and protein active site. This includes mapping of hydrogen-bonding sites in the ligand and protein. A least-square fitting process is employed to position the ligand within the active site of the protein in such a way that as many of the hydrogen bonds suggested by the mapping are formed upon decoding a chromosome. The fitness of a decoded chromosome is then a combination of number and strength of hydrogen bonds that are formed in this way in addition to the van der Waals energy of the bound complex.

\section{Preparation of the protein target and ligand}

Known crystal structures of oxy-Hemoglobin (Hb) (PDB Id: 1HHO) was obtained from the Brookhaven Protein Data Bank. The two dimensional (2D) structure of DOX (Figure 1) was downloaded from Pubchem (pubchem.ncbi.nlm.nih.gov). 2D to 3D conversion was done using CORINA [13]. Water molecules and ions were removed (including ordered water molecules) and hydrogen atoms were added at appropriate geometry. Groups within the protein were ionized as required at physiological $\mathrm{pH}$. The structure of $\mathrm{Hb}$ was protonated using Insight II (www.accelrys.com). The genetic algorithm was implemented in GOLDv3.1.1 that was applied to calculate the possible conformations of the drug binding to protein. The genetic algorithm parameters used in the analysis include, population size 100 , number of islands 5 , niche size 2 , selection pressure 1.1 , migrate 2; number of operators 100,000 , mutate 95 and crossover 95. A maximum of 10 different conformations was considered for the drug during the docking process. The conformer with the lowest binding free energy was used for further analysis.

\section{Molecular docking simulations}

All the conformations were then evaluated by X-Score. RMSD values of the best scored conformations of these protein-ligand complexes were calculated. The binding energy of docked complexes was calculated using X-Score [14]. The scoring functions have all the necessary elements that correspond to the non-covalent interactions in a conventional force field, such as the van der Waals interaction and the electrostatic interaction. It also considers the hydrophobic effect and thus provides a better estimation of binding free energies. A special feature is that three different algorithms have been implemented to calculate the hydrophobic effect term and this result in three parallel scoring functions. All three scoring functions are calibrated through multivariate regression analysis of protein-ligand complexes and this reproduces the binding free energies of the entire complex. These three scoring functions are further combined into a consensus scoring function called X-Score. The residues that are making hydrogen bonding and hydrophobic interactions were calculated using Getneares, a tool available with DOCKv5.1.1 [15].

\section{Discussion: \\ Hb drug binding}

The complementary applications of molecule modeling have been employed by computer methods to improve the understanding of the interaction of DOX and $\mathrm{Hb}$. The best docking result is shown in Figure 2. As predicted from the docking procedure the DOX binding site is situated on the alpha subunit of $\mathrm{Hb}$. The binding free energy $\left(\Delta \mathrm{G}^{\circ}\right)$ and the binding affinity (Ka) were found to be $-66.23 \mathrm{KJmol}^{-1}$ and $16.98 \mathrm{x}$ $10^{3} \mathrm{~mol}^{-1}$, respectively. Negative value of $\Delta \mathrm{G}^{\circ}$ shows that the binding reaction is thermodynamically favorable and the value of binding constant predicts high binding affinity between protein and drug.

\section{Binding site}

The amino acid residues involved in the binding of DOX to $\mathrm{Hb}$ were predicted and their respective molecular distances from the bound drug have been evaluated with the Getnears as presented in Table 1 (see supplementary material). The presented data revealed that His 45 is the closest residue to be found in the vicinity ( $5 \AA$ ) of the drug molecule and Gly57 was found to be far away. The modeling study showed that DOX binds to $\mathrm{Hb}$ on the surface binding site. At this site, the conformation of ligand differs from that in aqueous solution, where the mean plane of the sugar is perpendicular to the dihydroxyanthraquinone plane. In view of the finding that glycosidic linkage $(\mathrm{C}(6 \mathrm{a})-\mathrm{C}(7)$ $\left.\mathrm{O}(7)-\mathrm{C}\left(1^{\prime}\right)\right)$ has changed in DOX binding biomacromolecule [16], we can also predict the conformational change in $\mathrm{Hb}$ upon the binding of DOX molecule.

\section{Binding mode}

The validation of the binding mode as per the amino acid residue predicted to be part of the binding site is shown in Figure 2. The phenol ring of DOX is making hydrophobic interactions with Phe46, Ala53, Gly57. The interaction between $\mathrm{DOX}$ and $\mathrm{Hb}$ is not exclusively hydrophobic as there are several ionic and polar residues (His45, Gln54, Lys61, Lys90, and Arg197) in the proximity of the bound ligand (within $5 \AA$ ). These polar residues play an important role in stabilizing DOX via H-bonds and electrostatic interactions. Lys61 is in a suitable position involved in making $\mathrm{H}$-bonds with the imino groups of the side chain. Basic amino acids like Arg197 and Lys90 in the vicinity of DOX are targets for interactions with negatively charged carbonyl oxygen functions of the dihydroxyanthraquinone moiety. The hydrogen-bonding or electrostatic interaction acts as an anchor and this helps to attain the 3D space position of DOX in its binding pocket. This facilitates the hydrophobic interaction of the dihydroxyanthraquinone rings with the side chain of $\mathrm{Hb}$ residues. The DOX is in close proximity to the heme ring and this imply the interference in the oxygen binding property of the protein. It has been shown that the interaction of DOX with $\mathrm{Hb}$ induced a significant increase $(22 \%)$ in oxygen affinity as compared with $\mathrm{Hb}$ without drug [17]. 


\section{www.bioinformation.net}

Hypothesis

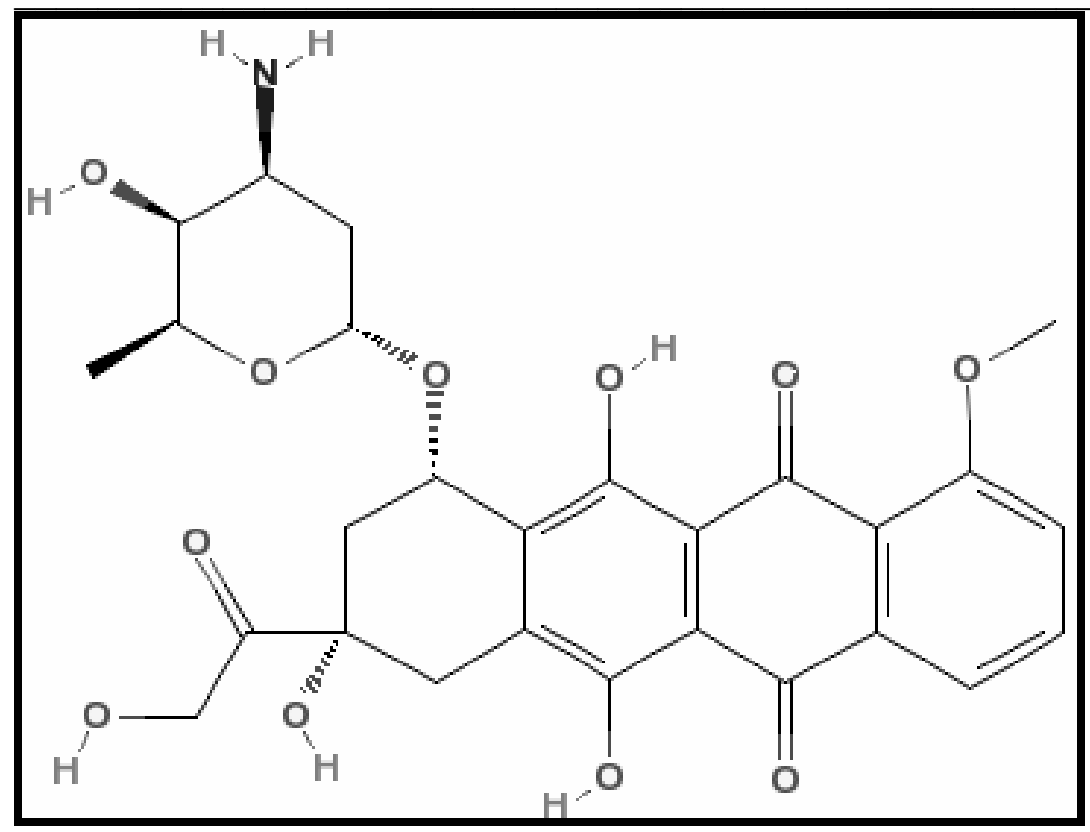

Figure 1: Two dimensional structure of doxorubicin downloaded from PubChem (pubchem.ncbi.nlm.nih.gov).

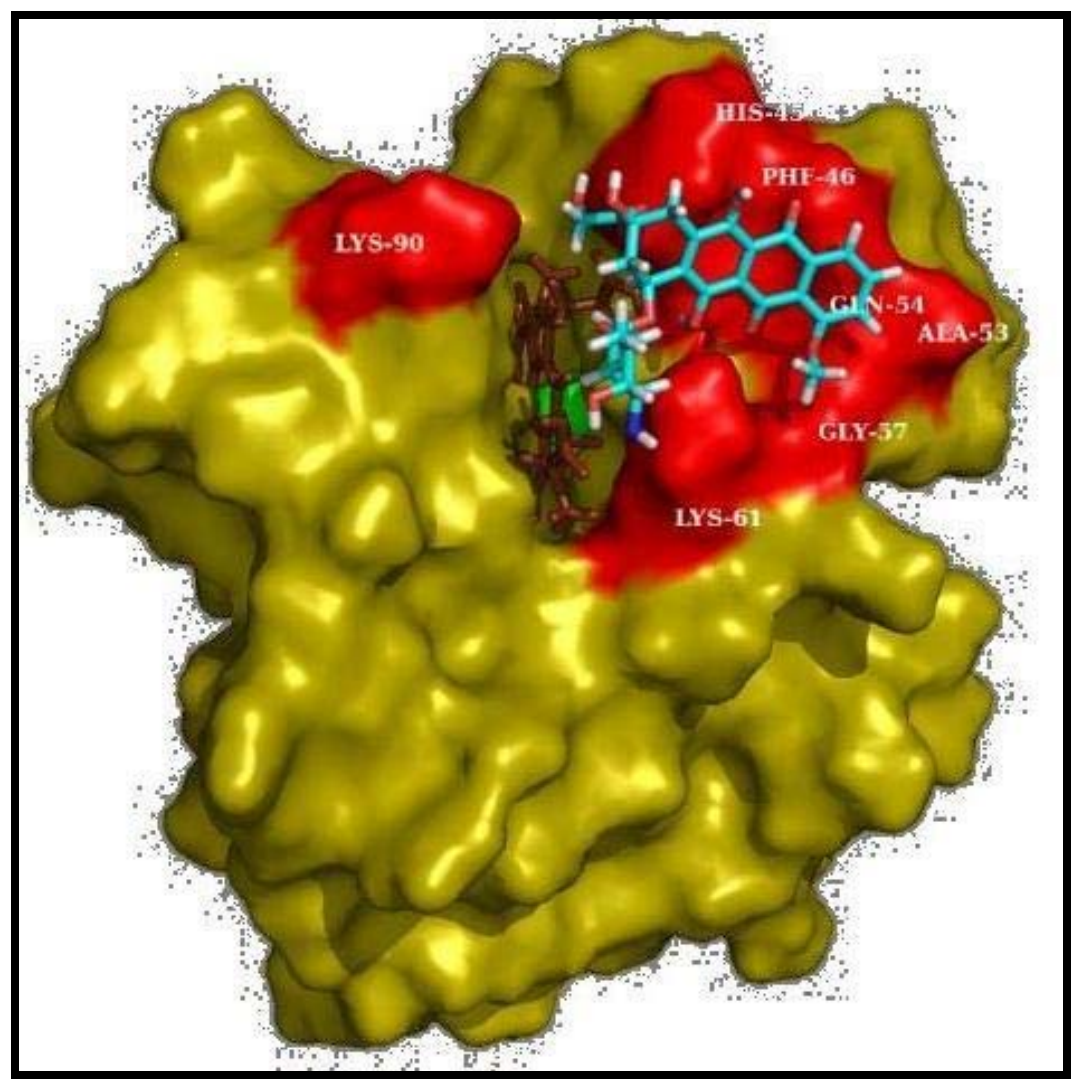

Figure 2: Doxorubicin docked onto hemoglobin zoomed to show the amino acid residues involved in the binding site. DOX, represented in stick (light blue) and hemoglobin, represented in surface colored in olive yellow. The heme ring depicted as brown color stick structure with bound oxygen (green). The image was prepared using Pymol (pymol.sourceforge.net). 


\section{Bioinformation}

\section{www.bioinformation.net}

\section{Hypothesis}

\section{Conclusion:}

The binding property of DOX with $\mathrm{Hb}$ has been studied using molecular surface analysis. The DOX binding properties of $\mathrm{Hb}$ was mapped using molecular docking techniques. Docking calculations found DOX to be located at the superficial site within the alpha subunit of $\mathrm{Hb}$. The model showed the microenvironment of DOX to be rich in polar (basic) amino acid residues able to stabilize the ligand. This work provides comprehensive insight and understanding for the molecular interaction of $\mathrm{DOX}$ with $\mathrm{Hb}$ in vivo.

\section{Acknowledgement:}

We are thankful to Prof. M. Saleemiddin, coordinator, IBU for providing us the facilities to carry out this work. The authors also acknowledge Dr. N. Subbarao, JNU, New Delhi, for his valuable guidance.

\section{References:}

[01] D. J. Naisbitt et al., Curr Opin Allergy Clin Immunol., 1: 317 (2001) [PMID: 11964707]

[02] N. R. Pumford and N. C. Halmes, Annu Rev Pharmacol Toxicol., 37: 91 (1997) [PMID: 9131248]

[03] U. Groth and H. G. Neumann, Chem Biol Interact., 4: 409 (1972) [PMID: 5030586]

[04] J. L. Miles et al., J Biol Chem., 237: 1319 (1962) [PMID: 14473857]
[05] F. W. Scheller et al., Adv Colloid Interface, 116: 111 (2005) [PMID: 16099417]

[06] K. Furuyama et al., Tohoku J Exp Med., 213: 1 (2007) [PMID: 17785948]

[07] S. De and A. Girigoswami, J of Colloid Interface Sci., 296: 324 (2006) [PMID: 16225884]

[08] F. M. Muggia and M. D. Green, Crit Rev Oncol Hematol., 11: 43 (1991) [PMID: 1831987]

[09] K. Shinohara and K. R. Tanaka, Hemoglobin, 4: 735 (1980) [PMID: 6254923]

[10] C. A. Henderson et al., Blood, 52: 878 (1978) [PMID: 698394]

[11] A. L. Sagone and G. M. Burton, Am. J. Hematol., 7: 97 (1979) [PMID: 539595]

[12] C. C. Winterbourn et al., Biochem. J., 179: 665 (1979) [PMID: 475774]

[13] http://www.mol-net.de

[14] R. Wang et al., J. Comput. Aided. Mol. Des., 16: 11 (2002) [PMID: 12197663]

[15] D. Kuntz et al., J Mol Biol., 161: 269 (1982) [PMID: 7154081]

[16] L. Gallois et al., Biochim. Biophys. Acta., 1370: 31 (1998) [PMID: 9518541]

[17] F. Misiti et al., Braz J Med Biol Res., 36: 1643 (2003) [PMID: 14666248]

Edited by V. S. Mathura

Citation: Khan and Khan, Bioinformation 2(9): 401-404 (2008)

License statement: This is an open-access article, which permits unrestricted use, distribution, and reproduction in any medium, for non-commercial purposes, provided the original author and source are credited.

\section{Supplementary material}

\begin{tabular}{lll}
\hline Protein atom & Ligand atom & Distance $(\AA)$ \\
\hline HIS 45 NE2 & 7 O7 DOX & 2.81 \\
PHE 46 CZ & 9 O9 DOX & 4.30 \\
ALA 53 O & 11 O11 DOX & 4.19 \\
GLN 54 CG & 38 C26 DOX & 3.43 \\
GLY 57 CA & 11 O11 DOX & 4.41 \\
LYS 61 NZ & 5 O5 DOX & 3.05 \\
LYS 90 NZ & 30 C18 DOX & 4.36 \\
HEM 1 O1D & 8 O8 DOX & 2.66 \\
\hline
\end{tabular}

Table 1: Molecular docking analysis of DOX binding to Hb. Prediction of atoms involved and estimated distances between protein and ligand atoms is given. 